W. STASZEK

\title{
ON THE CLASSICAL LOGIC OF NAMES
}

(Summary)

The paper contains a calculus of names with negation sign and quantifiers. This calculus is called the system $S$. Its primitive terms are: $\epsilon$ (is) and ' (negation sign).

The system $S$ has the following axioms:

A1. $\quad x \in x$

A2. $-x \in X^{\prime} \equiv \sim(x \in X)$

A3. $\quad-\left(\prod_{x}(x \in X \equiv x \in S) \cdot S \in P\right) \rightarrow X \in P$

A4. $\quad-\prod_{x}(x \in S \rightarrow x \in P) \rightarrow \prod_{x}(x \in P \rightarrow x \in S)$.

The sign "- " is to be read "is a theorem of the system $S$ ", whereas the constant " $\dashv$ " abbreviates the locution "is a rejected formula of the system $S$ ".

The well known two-termed predicates of Aristotle's syllogistic as well as the sign of equality are defined in usual way by means of the constant " $\epsilon$ " and quantifiers. Use is made of two kinds of proofs: the proofs of theorems of the system $S$ and, in addition, some special proofs of rejected formulae of $S$.

The following theorem is proved in the paper: Aristotle's syllogistic with the negation sign "' " is a decidable subsystem of the system $S$ in the following sense: for each well formed formula of Aristotle's syllogistic (with or without the sign "'") there exists a proof of the first or of the second kind. 